\title{
Radio Properties of GPS and CSS Sources: Galaxies versus Quasars
}

\author{
Carlo Stanghellini \\ Istituto di Radioastronomia del CNR, Contrada Renna, Noto, Italy, I-96017 \\ cstan@ira.cnr.it \\ Received 2002 July 2, accepted 2002 December 12
}

\begin{abstract}
On the basis of the different radio properties (especially different radio morphologies) between GPS galaxies and GPS quasars, I suggest that GPS galaxies are a more homogeneous class mainly consisting of compact symmetric objects, while the majority of GPS quasars are an intrinsically different type of radio source, similar to the common flat spectrum radio sources. For CSS radio sources the difference between galaxies and quasars is less evident. This is interpreted as a bias correlated with size: samples of smaller objects are more likely to be contaminated by unrelated objects. This is consistent also with the high percentage of blazar/quasar objects present in a sample of the even smaller HFP radio sources.
\end{abstract}

Keywords: galaxies: active — galaxies: evolution — quasars: general — radio continuum: galaxies

\section{Introduction}

Two basic models have been proposed to explain the subgalactic sizes of gigahertz peaked spectrum (GPS) and compact steep spectrum (CSS) radio sources.

The frustration model (van Breugel, Miley, \& Heckman 1984): the radio emitting plasma is permanently confined to the region within the host galaxy by an external medium which is dense enough to prevent the expansion of the radio source.

The youth model (Fanti et al. 1995; Readhead et al. 1996): based on the idea by Phillips and Mutel (1982) this model suggests that GPS sources are small because they are young; during the course of their life they will grow large and progressively decrease their radio luminosity by about one order of magnitude.

The different explanations of the nature of GPS/CSS radio sources, i.e. both the youth and the frustration scenarios, come from the morphological similarity between the small GPS/CSS galaxies and the extended radio galaxies.

The aforementioned models apply indeed only to the GPS radio galaxies. The GPS quasar phenomenon has been rarely taken into consideration. It is known that there is a clear difference in the distribution of redshifts between galaxies and quasars (Snellen 1997; Stanghellini et al. 1998). Moreover GPS galaxies and quasars also differ in other aspects. Galaxies have a symmetric radio morphology with sizes from a few parsecs to a few hundred parsecs while quasars have a core-jet or complex morphology (sometimes the core is weak or hidden) and smaller sizes (Stanghellini et al. 2001). GPS quasars have generally the turnover in the radio spectrum at a higher frequency than galaxies in both the observed and rest frame (Snellen 1997; Stanghellini et al. 1998).

Snellen (1997) has proposed a model in which the GPS galaxies seen along the jet axis should appear as flat spectrum sources, therefore GPS galaxies and GPS quasars should be objects of different classes. In any case, some authors consider also GPS quasars young radio sources whose non-thermal optical continuum is seen because of the orientation of the radio source, and put together GPS galaxies and GPS quasars.

\section{Morphological Differences and Extended Emission}

The detection of arcsecond scale faint extended emission around $\sim 10 \%$ of GPS sources (Baum et al. 1990; Stanghellini et al. 1990) motivated Baum et al. (1990) to suggest that nuclear activity is recurrent in these sources. In this hypothesis, we see the relic of a previous epoch of activity as faint diffuse emission surrounding the current young nuclear source.

I will discuss in detail a few objects of the complete sample of bright GPS radio sources selected by Stanghellini et al. (1998) where arcsecond scale extended emission has been detected, and I will try to make some inferences on the nature of GPS quasars. The images at arcsecond resolution are preliminary images from VLA B array data which will be extensively discussed in a paper in preparation, and the VLBI images are from Stanghellini et al. (1997, 2001), or are made from RRFID data kindly provided by Alan Fey ${ }^{1}$. Information given below on the optical hosts is taken from the NASA/IPAC Extragalactic Database $^{2}$.

0108+388: galaxy at $z=0.668$. This is a confirmed compact symmetric object (CSO) with a detected core (Taylor, Readhead, \& Pearson 1996). The radio galaxy $0108+388$ is a very interesting object because it has extended emission on the kpc scale but also a separation velocity of the micro-hot spots has been measured (Figure 1). Past AGN activity has been suggested to

\footnotetext{
${ }^{1}$ http://rorf.usno.navy.mil/rrfid.shtml

${ }^{2} \mathrm{http}: / /$ nedwww.ipac.caltech.edu
} 

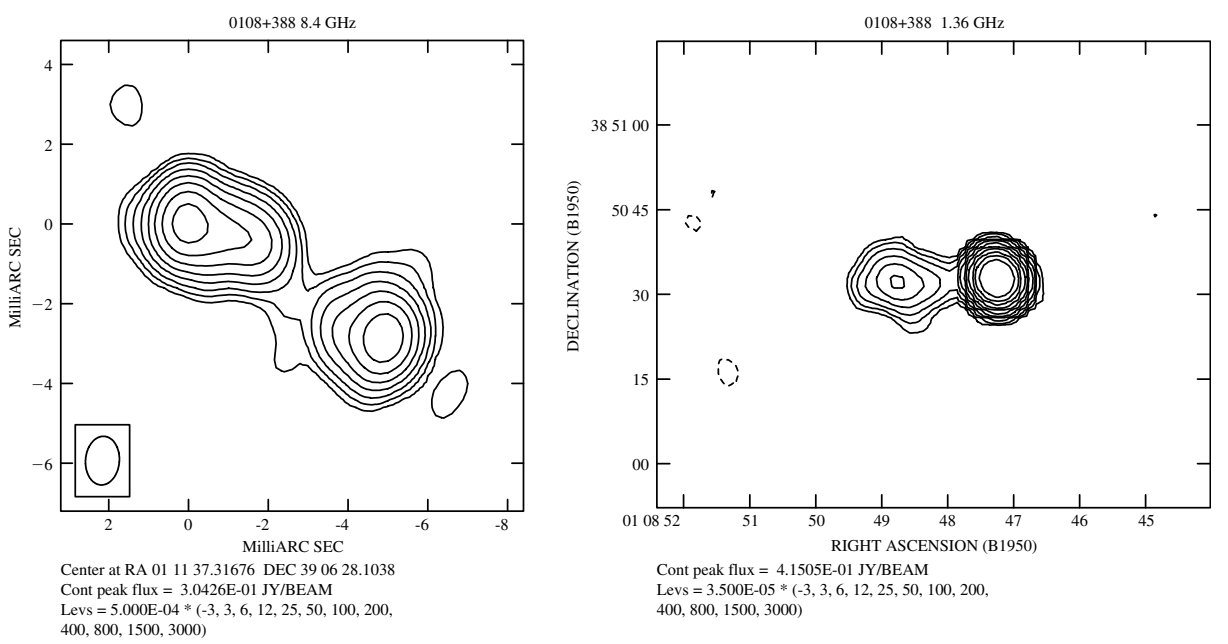

Figure $1 \quad 0108+388$ at mas and arcsecond resolutions.
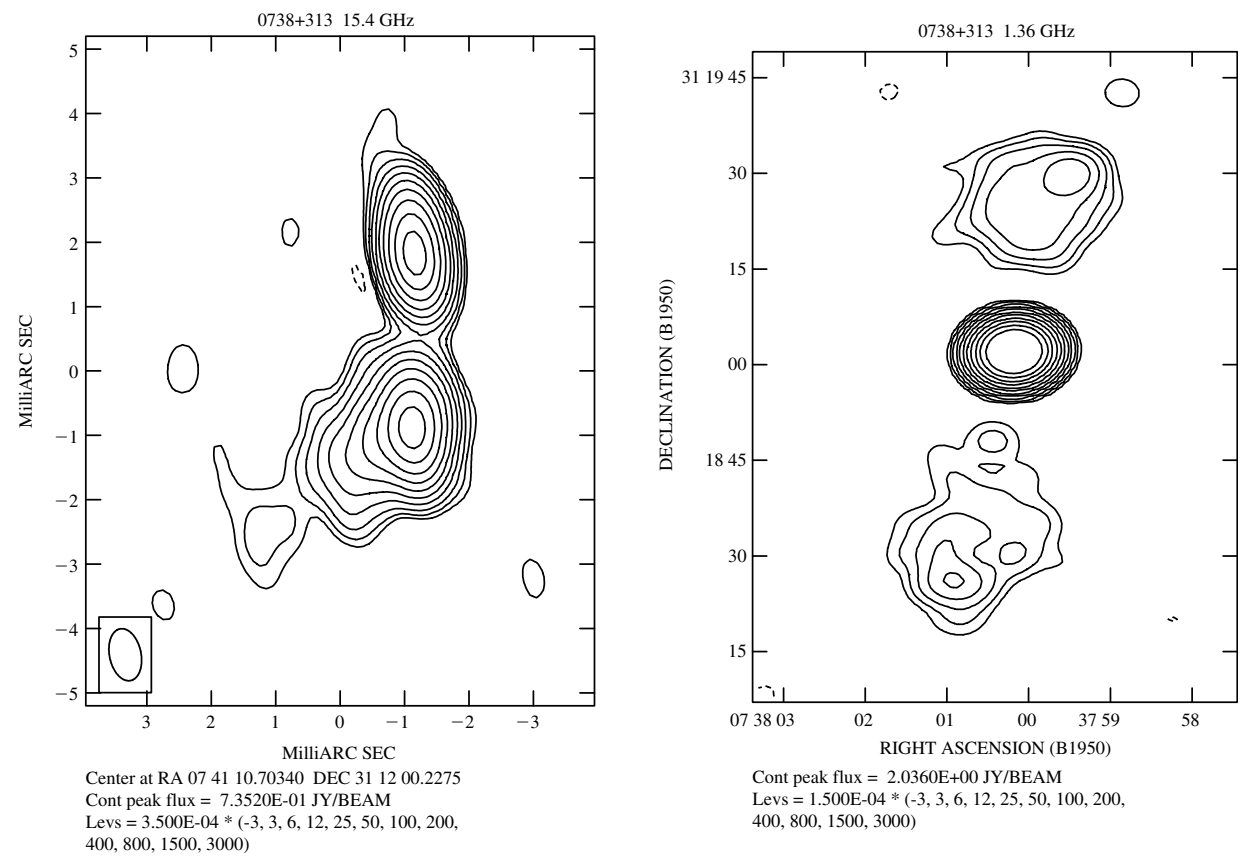

Figure $20738+313$ at mas and arcsecond resolutions.

explain the extended emission (Baum et al. 1990) in this object.

$0738+313$ : quasar at $z=0.635$. In $0738+313$ we see hot spots on an arcsecond scale which suggest the AGN is active and resupplying new electrons to the radio source at this arcsecond scale. We also see a mas morphology which is well explained by a core-jet structure (Figure 2) with a northern compact component with a flatter spectral index identified as the core (Stanghellini et al. 2001) and a jet showing a boosted knot where it changes direction (helical jet seen in projection?). This knot is likely responsible for the convex global radio spectrum.

1127-145: quasar at $z=1.187$. It is very likely this is a core-jet radio source at mas scale. The VLBI image of 1127-145 shows a rather compact western component (the core?), a more extended eastern component (a knot in a jet?), and a jet which, at the position of this 'knot', bends and continues to more or less the direction of the arcsecond scale jet (Figure 3). We do not see two hot spots at arcsecond scale as in $0738+313$, but the source is at a higher redshift and at arcsecond scale we may detect only part of the jet still moderately beamed toward us. Siemiginowska et al. (2002) report the detection of X-ray emission associated with this jet. The radio morphology is well explained by a jet which extends from the mas to the arcsecond scale.

1345+125: galaxy at $z=0.122$. This radio source is classified as a CSO on the basis of the presence of radio emission on both sides of the identified core (Xiang et al. 2002), even if the radio emission at the north of the core is much weaker than the southern counterpart. The arcsecond scale morphology of $1345+125$ resembles that of 

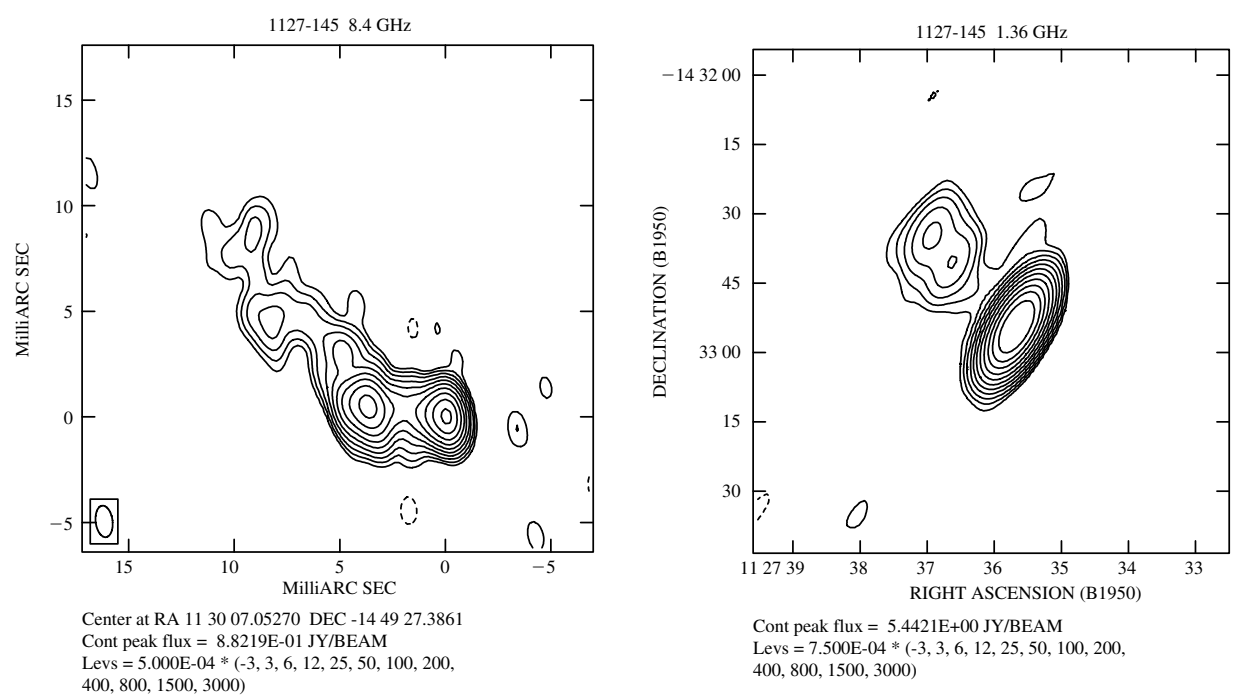

Figure $3 \quad 1127-145$ at mas and arcsecond resolutions.
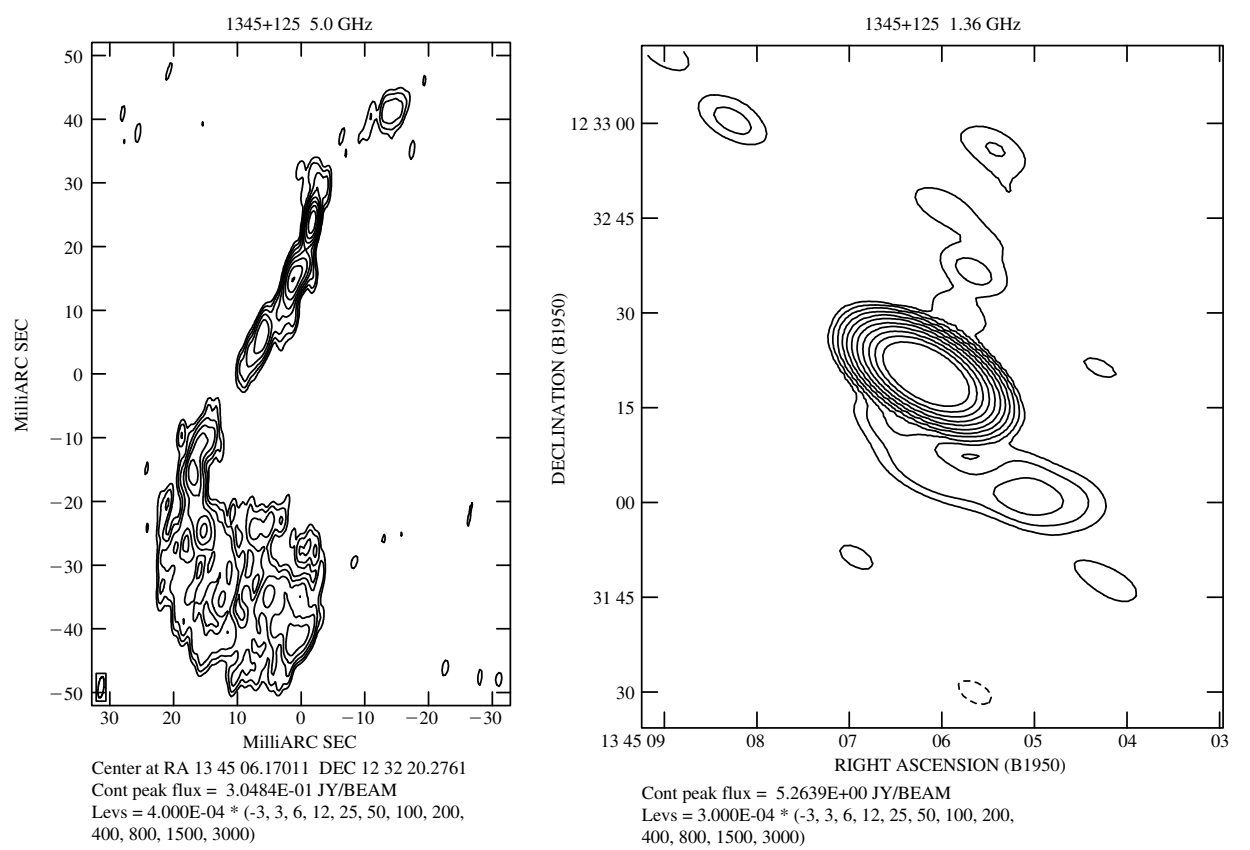

Figure $4 \quad 1345+125$ at mas and arcsecond resolutions.

the parsec scale: a southern region bending to the southwest, and a weaker, barely visible, northern component (Figure 4). In this case it is possible that the arcsecond scale radio emission is the relic of past activity of the radio galaxy, even if a continuous flow of plasma from the mas to the arcsecond scale cannot be excluded.

2134+004: quasar at $z=1.932$. The quasar $2134+004$ has a double morphology at mas scale which is, however, interpreted as a core-jet morphology, the core being the western, more compact component (Stanghellini et al. 2001). The comparison with the $8.4 \mathrm{GHz}$ image presented in Fey, Clegg, \& Fomalont (1996) indicates that the western component has a flatter spectral index, consistent with the above morphological classification. At arcsecond scales it shows extended emission around the dominant compact component (Figure 5; see also Stanghellini et al. 1998). Also in this case the simplest explanation for the radio emission at arcsecond scales is the presence of a jet seen in projection which extends from the mas to the arcsecond scale.

Taking into consideration all the GPS radio sources of the complete sample showing a clear presence of diffuse arcsecond scale emission around the compact component, only one case $(0108+388)$ is a good example of a radio source which has been reborn (in the framework of the youth scenario) or whose jets have been disrupted (in the framework of the frustration scenario). The radio galaxy $1345+125$ may also be explained in such a way, but in the other three cases (all quasars) presented here the most natural explanation is that we see a large radio source where 

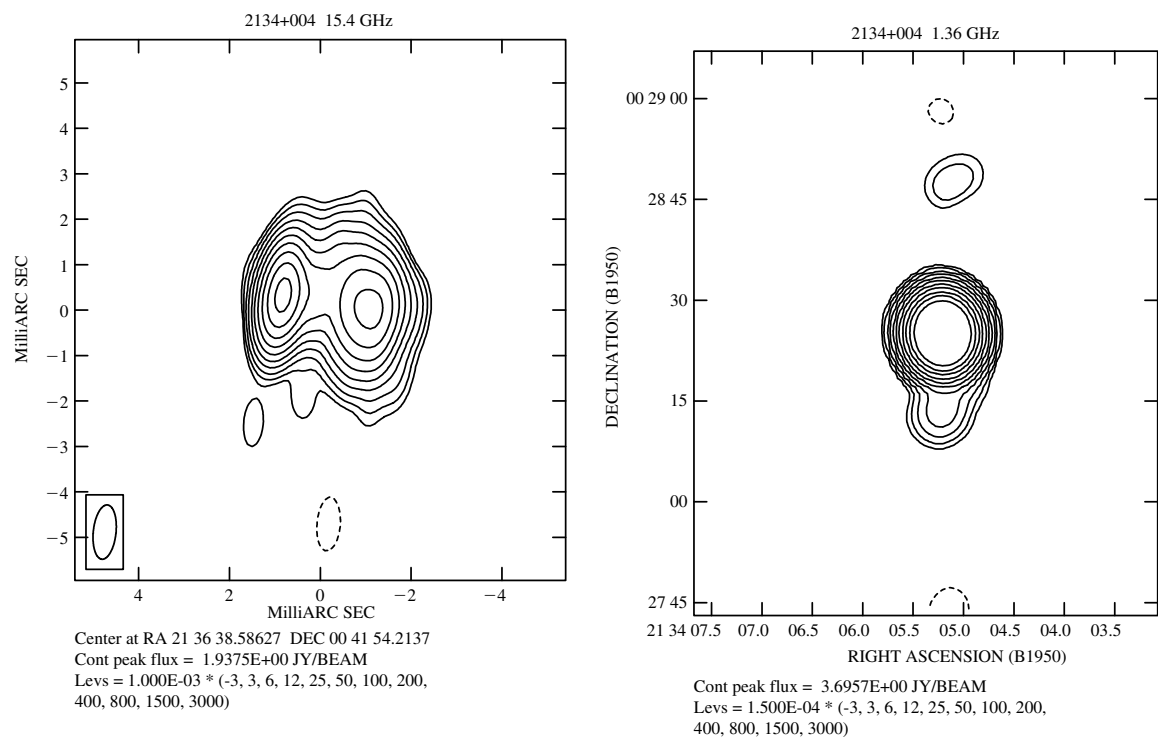

Figure $52134+004$ at mas and arcsecond resolutions.

the inner part of the jet is boosted because it is pointed toward us. If we also consider the redshift distribution of the GPS quasars we see that extended emission at the level of that detected around $0738+313$ (which is the closest quasar of the sample) would be too weak to be detected for most of the other quasars in the sample. Therefore we can speculate that the GPS quasars showing a core-jet or complex morphology at mas scales are mostly large radio sources at high redshift where only the inner part of the jet is visible due to relativistic and projection effects. If this is true, most GPS quasars are intrinsically more similar to the common flat spectrum radio sources which dominate the radio surveys at $\mathrm{cm}$ wavelengths, and are not related to the CSO phenomenon.

\section{The Radio Flux Density Variability and Polarisation}

It is often stated in the literature that GPS radio sources have little variability and do not show superluminal motions, the relativistic effect often seen in flat spectrum radio sources. These characteristics are attributed to the GPS class without distinction of the host (galaxy or quasar). However, these two properties are based on the lack of a systematic study, since there are no flux density monitoring campaigns on large samples of GPS/CSS sources.

In the literature information can be found on flux density variability regarding several objects belonging to the complete sample. Some sources, such as 0237-233, $1245-197$, and $1345+125$, were found to be stable. On the other hand 1127-145 and 2134+004 were found to be significantly variable (Stanghellini et al. 1998, and references therein).

In order to test the general behaviour of GPS radio sources in terms of flux density variability, the flux density measurements have been compared for the objects in common between the complete sample (for details see
Stanghellini 1999) and the sources observed by Perley (1982). For the galaxies the mean variability has been found to be around 5\% while for the confirmed GPS quasars it is $\sim 20 \%$. For a comparison sample of flat spectrum radio sources the mean variability has been found to be $\sim 22 \%$, thus with respect to the variability, GPS and non-GPS quasars seem to have similar 'mean' behaviour.

Another common characteristic attributed to the GPS class is the very low radio polarisation. Indeed the GPS galaxies of the complete sample are undetected at $6 \mathrm{~cm}$ by the VLA below $0.3 \%$ and many below $0.1 \%$. The GPS quasars instead have a mean fractional polarisation at $6 \mathrm{~cm}$ of $1.2 \%$, while for a comparison sample of nonGPS quasars I found 1.8\% (Stanghellini 1999). Therefore there is some evidence that GPS quasars have a lower fractional polarisation than non-GPS quasars but they are significantly more polarised than GPS galaxies.

\section{Samples of CSS and HFP Radio Sources}

GPS radio sources are almost evenly optically identified with galaxies and quasars in the complete sample selected by Stanghellini et al. (1998). What happens if we look at other samples?

Dallacasa et al. (2000) selected a sample of extreme GPS radio sources with convex radio spectra peaking at frequencies above a few $\mathrm{GHz}$, and with a flux density limit at $5 \mathrm{GHz}$ of $300 \mathrm{mJy}$. They call these radio sources high frequency peakers (HFPs). That sample extends to higher turnover frequencies the samples of CSS and GPS radio sources. Dallacasa, Falomo, \& Stanghellini (2002a) discuss the optical identification of the objects in the sample and they find that $26 \%$ are galaxies and $74 \%$ are quasars or stellar objects.

Fanti et al. (2001) selected a new sample of low/medium brightness CSS radio sources from the B3 sample. Dallacasa et al. (2002b, 2002c) observed 46 of them with MERLIN, EVN, and VLBA. They find that 
36 are CSOs or MSOs (medium-size symmetric objects), two have core-jet, and eight have uncertain or irregular morphology.

Thus most of the bright HFPs are blazars/quasars, half of the bright GPS sources are core-jet quasars, and only some faint CSS sources are core-jet objects. It seems that the incidence of quasars and/or core-jet morphologies decreases with decreasing turnover frequency (i.e. increasing size), and possibly decreasing brightness.

This is consistent with the view that the GPS spectrum in quasars and galaxies originates from intrinsically different emitting regions: micro-lobes/hot spots with a large range in sizes in galaxies, while in quasars the emitting region tends to be more compact and close to the core.

\section{What are GPS Quasars?}

Given the above results, it is likely that most GPS quasars (generally showing core-jet or complex mas structures) are intrinsically similar to the flat spectrum radio sources, and we see a convex radio spectrum because a single rather homogeneous component (maybe a knot in a jet) dominates the radio emission.

Some GPS quasars can still be young/frustrated/recurrent radio sources seen with the AGN axis more aligned toward us, but in any case it is the mas morphology which should discriminate. If there is any indication of microlobes or micro-hot spots then they are likely to be truly small. If we do not see a CSO morphology, a large and old radio source seen along the radio axis is the more simple (and likely) explanation. Even if we do not see detectable extended emission it is possible that this radio emission is well below the detection limit because of the high redshift of GPS quasars.

The GPS quasars could be those objects where the radio emission is not dominated by the core alone, but exhibit a significant amount of emission from one or a few knots in a moderately beamed jet. These knots may correspond to the place of a shock front where the electrons are re-accelerated, or a place in an helical jet where the bulk flow is directed toward the observer enhancing its brightness by moderate relativistic beaming.

In short, GPS samples are contaminated by radio sources (mostly quasars) that have nothing to do with the youth/frustrated/recurrent dilemma.

The selection should be based on the mas morphology rather than on the global radio spectrum only. Indeed it is easier to select sources on the basis of the radio spectrum because there are several all-sky surveys at different frequencies, while VLBI surveys are much more limited. But, after having done the radio spectral selection, the contamination should also be taken into serious consideration. Or, in other words, the nature of the (majority of?) GPS quasars should be studied separately.

\section{Conclusions}

(i) Arcsecond scale emission around CSO/GPS galaxies is well explained as the co-presence of past and new radio activity, like in $0108+388$ and (maybe) $1345+125$. (ii) GPS quasars showing extended emission are likely to be large radio sources seen in projection, like $0738+313$, 1127-145, and 2134+004. (iii) Extended emission is possibly not detectable at high redshift. (iv) GPS quasars are indeed more variable in radio flux density and more polarised than commonly assumed, and do not differ too much in this respect from flat spectrum radio sources. (v) Quasars with core-jet or complex morphology tend to be more common in samples of brighter and smaller radio sources, dominating in number the HFP sample, and being only a few in the low brightness CSS sample.

Therefore there is strong evidence that GPS/CSS quasars with a core-jet/complex mas morphology and CSO sources are different classes of objects. Any inference based on complete samples of GPS/CSS radio sources should take into consideration this contamination and should be limited to CSO sources when information on morphologies is available, or limited to galaxies if mas morphologies are unknown.

\section{Acknowledgments}

This research has made use of the United States Naval Observatory (USNO) Radio Reference Frame Image Database (RRFID).

\section{References}

Baum, S. A., O’Dea, C. P., de Bruyn, A. G., \& Murphy, D. W. 1990, A\&A, 232, 19

Dallacasa, D., Stanghellini, C., Centonza, M., \& Fanti, R. 2000, A\&A, 363, 887

Dallacasa, D., Falomo, R., \& Stanghellini, C. 2002a, A\&A, 382, 53

Dallacasa, D., Tinti, S., Fanti, C., Fanti, R., Gregorini, L., Stanghellini, C., \& Vigotti, M. 2002b, A\&A, 389, 115

Dallacasa, D., Fanti, C., Giacintucci, S., Stanghellini, C., Fanti, R., Gregorini, L., \& Vigotti, M. 2002c, A\&A, 389, 126

Fanti, C., Pozzi, F., Dallacasa, D., Fanti, R., Gregorini, L., Stanghellini, C., \& Vigotti, M. 2001, A\&A, 369, 380

Fanti, C., Fanti, R., Dallacasa, D., Schilizzi, R. T., Spencer, R. E., \& Stanghellini, C. 1995, A\&A, 302, 317

Fey, A. L., Clegg, A. W., \& Fomalont, E. B. 1996, ApJS, 105, 299

Perley, R. A. 1982, AJ, 87, 859

Phillips, R. B., \& Mutel, R. L. 1982, A\&A, 106, 21

Readhead, A. C. S., Taylor, G. B., Xu, W., Pearson, T. J., Wilkinson, P. N., \& Polatidis, A. G. 1996, ApJ, 460, 612

Siemiginowska, A., Bechtold, J., Aldcroft, T. L., Elvis, M., Harris, D. E., \& Dobrzycki, A. 2002, ApJ, 570, 543

Snellen, I. A. G. 1997, PhD Thesis, University of Leiden

Stanghellini, C. 1999, MmSAI, 70, 117

Stanghellini, C., Baum, S. A., O'Dea, C. P., \& Morris, G. B. 1990, A\&A, 233, 379

Stanghellini, C., O'Dea, C. P., Baum, S. A., Dallacasa, D., Fanti, R., \& Fanti, C. 1997, A\&A, 325, 943

Stanghellini, C., O'Dea, C. P., Dallacasa, D., Baum, S. A., Fanti, R., \& Fanti, C. 1998, A\&AS, 131, 303

Stanghellini, C., Dallacasa, D., O'Dea, C. P., Baum, S. A., Fanti, R., \& Fanti, C. 2001, A\&A, 377, 377

Taylor, G. B., Readhead, A. C. S., \& Pearson, T. J. 1996, ApJ, 463,95

van Breugel, W., Miley, G., \& Heckman, T. 1984, AJ, 89, 5

Xiang, L., Stanghellini, C., Dallacasa, D., \& Haiyan, Z. 2002, A\&A, 385,768 